\title{
Histochemical study of the pre- and postnatal develop- ment of acetylcholinesterase in the rat spinal cord
}

\author{
ZHANG QIN, $\mathrm{X}_{\text {INWEN DONG }}$ \\ Laboratory of neuromorphology, Department of Develop- \\ mental Neurobiology, Shanghai Institute of Physiology, \\ Academia Sinica, Shanghai 200031, China
}

\begin{abstract}
The distribution of acetylcholinesterase (AChE)positive structures in the developing rat spinal cord was studied with AChE-histochemistry. AChE-positive perikarya were first seen on embryonic day 14 (E14) in the ventrolateral portion of the spinal cord. From that time onward, AChE-containing cells appeared gradually in the intermediate gray, dorsal horn and lateral spinal nucleus of the spinal cord in a ventral-to-dorsal, and lateral-tomedial order. No obvious rostral-to-caudal sequence was found. At birth, the distribution pattern of AChE-positive perikarya was basically similar to that in adults. After birth a dramatic increase in the AChE staining intensity extended from postnatal day 5 (P5) to postnatal day 21 (P21). In addition, two phases of transient AChE staining were observed in the external surface of the dorsal horn from embryonic day 15 (E15) to embryonic day 21 (E21) and in the marginal layer from embryonic day 21 (E21) to postnatal day 14 (P14), respectively.
\end{abstract}

Key words: Development, acetylcholinesterase, rat, histochemistry, spinal cord.

\section{INTRODUCTION}

Both biochemical and histochemical studies have shown that AChE is mainly present in the gray matter of adult spinal cord [1-3]. Biochemical assays[4-6] have

1. Corresponding author 
demonstrated that AChE appears at early fetal life and the increased AChE activity in later fetal life has been correlated with the morphological development of neurons in the embryonic spinal cord. To our knowledge, histochemical study of the ontogeny of AChE-containing cells in the spinal cord is very scarce, except a brief report presented by Kasa[5], although histochemical analysis has some advantages that biochemical analysis does not have. For a better understanding of the development of the AChE-containing neurons, we have attempted to elucidate the ontogenetical development of this enzyme in the rat spinal cord using the AChE histochemical method.

\section{MATERIALS AND METHODS}

Male and female Sprague-Dawley rats were caged together overnight and vaginal smears were analyzed on the following morning, the day of positive sperms was designated as embryonic day 1 (E1). Birth usually occurred on E22 or E23. At least 6 specimens obtained from three litters were examined from each of the following times: E12, E13, E14, E15, E16, E17, E18, E19, E21, postnatal day (P)1, P3, P5, P7, P10, P14, P20, P30 and P90 (adult). One third of the postnatal rats were pretreated with diisopropylfluorophosphate (DFP) $(1 \mathrm{mg} / \mathrm{kg}$ injected subcutaneously for P1-P14 rats, $2 \mathrm{mg} / \mathrm{kg}$ intramuscularly for P20-P90 rats) 10-12 h before perfusion. Fetuses of 17 days gestation or less were removed from the uterus and immediately immersed in ice cold $\left(4^{\circ} \mathrm{C}\right)$ 4\% paraformaldehyde in $0.1 M$ phosphate buffer $(\mathrm{pH}$ 7.4) for $5 \mathrm{~h}$ or dissected out following rapid transcardial perfusion of the pregnant dam with fixative and immersed into a further solution of the same fixative for 3-5 h. Fetuses of E18, El9, E21 and postnatal pups were perfused through the heart with the same fixative and then postfixed for 2-3 h. Following fixation, the rat spinal cord was transferred into $0.1 \mathrm{M}$ phosphate buffer containing $20 \%$ sucrose overnight. Coronal sections at 20 $\mu \mathrm{m}$ were cut on a cryostat and the sections mounted on gelatin coated slides. Most specimens were stained for AChE using the Mesulam modification of Karnovsky and Roots method[7]. The sections were first washed in $0.01 M$ PBS and incubated at room temperature for $3 \mathrm{~h}$ in a medium $(\mathrm{pH} \mathrm{5.0)}$ containing $4 \mathrm{~m} M$ acetylthiocholine iodide, $10 \mathrm{~m} M$ glycine, $2 . \mathrm{mM}$ copper sulfate, $50 \mathrm{~m} M$ sodium acetate and $0.2 \mathrm{~m} M$ ethopropazine hydrochloride. After incubation, the sections were rinsed in 0.1 $M$ acetate buffer ( $\mathrm{pH}$ 6.0) and then reacted in 10\% potassium ferricyanide solution for 2.5 min. washed in acetate buffer, and routinely converslipped. At each age before P5 two specimens were also processed for AChE histochemistry according to Karnovsky and Roots method as modified by Tago et al[8]. Sections were pretreated with $0.1 \%$ hydrogen peroxide for 30 min before staining procedure, then rinsed in maleate buffer $(\mathrm{pH}$ 6.0) and incubated for 2-3 $\mathrm{h}$ in Karnovsky and Roots medium at a dilution of 1:50. After extensive rinsing, the sections were incubated for $5 \mathrm{~min}$ in a solution containing $0.04 \% 3,3$-diaminobenzidine and $0.3 \%$ nickel ammonium. Then a solution of $\mathrm{H}_{2} \mathrm{O}_{2}$ was added to a final concentration of $0.003 \%$ and sections incubated for another $10 \mathrm{~min}$.

At each age, separate sets of sections for controls were reacted in the presence of $10^{-5} \mathrm{M}$ eserine salicylate which selectively inhibits cholinesterase or with omission of the acetylthiocholine substrate.

\section{RESULTS}

No remarkable differences between the two AChE staining protocols used in this study could be detected. The Mesulam modification of Karnovsky and Roots method permitted us to observe the variations of the AChE staining intensity, whereas the sections stained according to Tago et al procedure enabled us to take clear black and white photographs. 
No AChE-positive staining was detected in the spinal cord before E14. However, at E12 and E13, AChE-positive staining was observed in the dorsal root ganglions and the dorsal part of boundary cap. At E14, very faintly stained AChE neurons were first detected in the lateral portion of the ventral horn throughout the length of the spinal cord (Plate I A). At this stage, the shapes of the AChE-positive cells could hardly be clearly delineated. On E15, the AChE-positive cells extended dorsally into the intermediolateral nucleus of the thoracolumbar segment (Plate I B ). By E17, the $\mathrm{AChE}$-positive structures identified at the foregoing stages increased in cell number, yet were still weakly stained. At this stage, a small number of AChE-positive cells were newly found in the medial portion of the ventral horn and the intermediomedial nucleus (Plate I C). At E19 the staining intensity of AChE in the ventral horn and intermediolateral nucleus increased, and the AChE reaction product could be traced into the proximal axons of the motoneurons (Plate I D). In addition, a narrow $\mathrm{AChE}$-positive band formed between intermediolateral nucleus and intermediomedial nucleus, and newly appeared AChE-positive cells were visualized in the central gray matter (Plate I E). At E21 and P1, AChE-positive cells made their appearance in the dorsal horn (layers I-IV), lateral cervical nucleus and lateral spinal nucleus (Plate I F, Plate II B). During this period, the delimitations of AChE-positive cells began apparent (Plate I F, Plate II A). At P7, the superficial layers (layers I and II) of the dorsal horn acquired a faintly stained AChE band and the neuropil in the spinal cord was also stained weakly (Plate II E). Thereafter, there was a dramatic increase in the staining intensity of both AChE-positive perikarya and neuropil. At P10, the AChE-positive band in the dorsal horn could be readily observed (Plate III F). At this stage the whole spinal cord gained a widespread staining, it was difficult to distinguish the AChE-positive cell bodies from fibers in non-DFP-treated specimens. It was seen that the increase in AChE staining intensity was chiefly due to the increase in neuropil through comparison between the DFP-pretreated specimens and non-DFP-treated specimens (Plate III F, G, H). However, the AChE-positive perikarya in the dorsal horn increased only slightly in their staining intensity and retained the weak staining until adulthood. By P20, the distribution pattern and staining intensity of AChE-positive structures in the spinal cord were almost similar to those seen in adult. (Plate III J, K).

Two phases of transient AChE staining in the ontogeny of the spinal cord were observed in the present study. At E15, the AChE-positive structure that was originally found in the position of the dorsal part of boundary cap shifted medially along the external surface of the dorsal horn (Plate I B). By E17, it formed an AChE-positive band on the surface of the dorsal horn (Plate I C). At E18, this band had completely ingressed into the dorsal funiculus (Plate I D, E). By E21 to P1, this AChE-positive staining band became undetectable histochemically (Plate I F). Almost at the same time, another transient AChE-positive staining appeared in the marginal layer of dorsal horn (Plate II B). Unlike other AChE-positive cells in the spinal cord that gained a discernible increase in staining intensity about one week after appearance, 
Development of AChE in the rat spinal cord

these cells were stained moderately for AChE once occurred. Their staining intensity did not change markedly during the period of their existence. But these cells were heterogeneous in cellular density from clustered cells to solitary cell (Plate II B-E, Plate III F-H), which could be found among pups and at different levels of the spinal cord in the same pup. At P14, the transient AChE-positive cells began to decrease in number (Plate III), and by P21, disappeared completely.

\section{DICUSSION}

The present study demonstrated that AChE-positive cells in rat spinal cord appeared progressively in a ventral-to-dorsal, lateral-to-medial order during the ontogeny, but no obvious rostral-to-candal order could be found. These results were in agreement with other studies concerned with neurogenesis and physiological development. Studies on neurogenesis[9-13] have well shown that the spinal cord develops in a ventral-to-dorsal order, with motoneurons being the first cells to occur, followed by interneurons, and finally the small nerve cells of the dorsal horn. Physiological development follows a similar sequence. It has been shown that spontaneous motility occurs earlier than reflexogenic movements[14,15]. However, there remains a dispute about the sequence of neuronal differentiation between the lateral motoneurons and the medial ones. Altman and Bayer[9] showed that the lateral motoneurons were developed before the medial ones both quantitatively and qualitatively. However, Fujita[10] showed a reverse sequence that the medial motoneurons are born first, and still other studies[12] showed no significant difference in the generation sequence between medial and lateral motoneurons. In the present work, the resuits seemed to support the lateral-to-medial development gradient based on the observation that the cells demonstrated $\mathrm{AChE}$ reaction product in a temporal sequence similar to that of differentiation. It has been shown by Altman and Bayer that a rostral-to-caudal gradient existed in the production of motoneurons[9]. This gradient, however, was not found in the study presented here. One cause of the discrepancy may be that the magnitude of the temporal differences at different levels is so small (only one day) that the differences in the appearance of AChE were not detectable appreciably by histochemical method.

It has been reported that AChE could be detected biochemically very early in ontogenesis[4,6], as early as at E9 reported by Kasa[5]. After its occurrence in the spinal cord, AChE showed a steady increase in enzyme activity until P21. In contrast to the biochemical assays, the present study showed the histochemically detectable AChE activity first occurred at E14. The difference between biochemical and histochemical results may be due to the partial inactivation of the enzyme activity by histochemical process. An alternative interpretation is that the spinal cord samples used in biochemical analysis may include the dorsal root ganglia and boundary-caps which showed AChE positive reaction product earlier than the spinal cord as a whole. According to the present work, the increase in AChE 
activity before P5 is mainly contributed by perikarya, and from P5 to P21 chiefly by neuropil. In addition, Phelps et al[16] have studied the postnatal development of choline acetyltransferase (ChAT)-immunoreactive cells of the spinal cord in which they showed that at birth the somatic and visceral motoneurons gained a moderate ChAT-immunoreactive staining, while the ChAT-immunoreactive cells in the central gray matter and dorsal horn were stained only weakly. The ChAT-immunoreactive intensity gradually increased up to P21 when these cells acquired adult-like staining property. A comparison between the results of Phelps et al's and ours showed the two main elements of cholinergic system in the spinal cord developed at a similar pace at least after birth.

In addition to confirming the transient $\mathrm{AChE}$ expression in the marginal layer cells first described by $\mathrm{K}$ asa[5] with more details and some differences, the present study also demonstrated another transient AChE expression which is related with fibers. Besides, the transient AChE appearance during ontogenesis has also been observed in thalamus[17], visual cortex[18], auditory cortex[20] and so on. Even though some functions other than acetylcholine degradation have been proposed for AChE in the developing nervous system[20], no direct evidence has yet been available to define its role clearly in ontogenesis. The transient expression on AChE in somatostatin-like immunoreactive neurons in stratum orients of the mouse hippocampus showed by Forloni et al[21] and co-expression of transient ChAT-immunoreactivity and AChE in Purkinje cells of the rat cerebellum demostrated by Gould and Butcher[22] have thrown some light on this problem. Since the transient AChE staining appears in various areas of the central nervous system, only one explanation about its role in the development is not adequate. We think that the transient appearance of AChE in the external surface of the dorsal horn and the dorsal funiculus may be related with the morphogenetic maturation of the fasciculi cuneatus and gracilis, perhaps unrelated to the growth guidance of these fibers, because the shift process to medial aspect of this transient AChE staining always occurs later than the growth of dorsal funiculus. Altman and Bayer have reported that the dorsal funiculus ascending zone appeared at E17 and the growth of ascending dorsal funiculus fibers is largely completed on E18[9]. With regard to the transient expression of AChE in the marginal layer, it may be reasonable to consider it as a developmental vestige, because of its heterogeneity in distribution among the pups and different levels of the same pup.

\section{ACKNOWLEDGEMENTS}

This work was supported by the National Natural Science Foundation of China. The authors wish to thank Ms. Shan Hong Ying for technical assistance.

\section{REFERENCES}

[1] K'asa P. The cholinergic systems in brain and spinal cord. Prog neurobiol 1986; 26:211-72.

[2] Marchand R, Barbeau H. Vertically oriented alternating acetylcholinesterase rich and poor terri- 


\section{Development of AChE in the rat spinal cord}

tories in laminae VI, VII, VIII of the lumbosacral cord of the rat. Neuroscience 1982; 7:1197-202.

[3] Navaratnam V, Lewis PR. Cholinesterase-containing neurons in the spinal cord of the rat. Brain Res 1970; 18:411-25.

[4] Burt AM. Choline acetyltransferase and acetylcholinesterase in the developing rat spinal cord. Exp Neruol 1975; 47:173-80.

[5] Kasa P. Development of the spinal cord and the acetylcholine system. In: Lissak K editor. Results in Neuroendocrinology, Neurochemistry and Sleep Research, Vol 7. Budapest: Hung. Acad Sci 1978:145-68.

[6] Maletta GJ, Vernadakis A, Timiras P S. Pre - and post -natal development of the spinal cord: increased acetylcholinesterase activity. Proc Soc Exp Biol(NY) 1966; 121:1210-1.

[7] Mesulam M-M. A horseradish peroxidase method for the identification of the efferents of acetylcho -linesterase-containing neurons. J Histochem Cytochem 1976; 24:1281-6.

[8] Tago H, Kimura H, Maeda T. Visualization of detailed acetylcholinesterase fiber and neuron staining in rat brain by a sensitive histochemical procedure. J Histochem Cytochem 1986; 34:1431-8.

[9] Altman J, Bayer SA. The development of the rat spinal cord. Adv in Anat, Embryol and Cell Biol Vol 85. Berlin: Springer-Verlag 1984.

[10] Fujita S. Analysis of neuron differentiation in the central nervous system by tritiated thymidine autoradiography. J Comp Neurol 1964; 122:311-27.

[11] Nornes HO, Das GD. Temporal pattern of neurogenesis in spinal cord of rat, I. An autoradiographic study- time and sites of origin and settling patterns of neuroblast. Brain Res 1974; 73:121-38.

[12] Phelps PE, Barker RP, Vaughn JE. Generation patterns of four groups of cholinergic neurons in rat cervical spinal cord: a combined tritiated thymidine autoradiographic and choline acetyltransferase immunocytochemical study. J Comp Neurol 1988; 273:459-72.

[13] Sims TJ, Vaughn JE. The generation of neurons involved in an early reflex pathway of embryonic mouse spinal cord. J Comp Neurol 1979; 183:707-20.

[14] Narayanan CH, Fox MW, Hamburger V. Prenatal development of spontaneous and evoked activity in the rat (Rattus nervergicus albinus). Behaviour 1971;40:100-33.

[15] Saito K, Ito S, Seno N, Kitazawa T: Ohga A. Development of spinal reflexes in the rat fetus. In: Goldberger ME, Gorio A, Murray M, editors. Development and plasticity of the mamma lian spinal cord. Padova: Liviana Press 1986:25-38.

[16] Phelps PE, Barker RP, Houser CR, Crawford GD, Salvaterra PM, Vaughn JE. Postnatal development of neurons containing choline acetyltransferase in rat spinal cord: an immunocytochemical study. J Comp Neurol 1984; 229:374-61.

[17] Kristt DA. Acetylcholinesterase in the ventrobasal thalamus: transience and patterning during ontogenesis. Neurosciences 1983; 10:923-39.

[18] Pobertson RT, Haines MA, Yu J. Investigation of the origins of transient acetylcholinesterase activity in developing rat visual cortex. Dev Brain Res 1988; 41:1-23.

[19] Robertson RT, Mostamand F, Kageyama GH, Gallardo KA, Yu J. Primary auditory cortex in the rat: transient expression of acetylcholinesterase activity in developing geniculocortical projections. Dev Brain Res 1991; 58:81-95.

[20] Silver A. The significance of cholinesterase in the developing nervous system. Prog Brain Res 1971; 34:345-55.

[21] Forloni G, Blake K, Hohmann CH, Coyle JT. The postnatal expression of acetylcholinesterase in somatostatin-positive cells of mouse hippocampus. Dev Brain Res 1989; 48:73-85.

[22] Gould E, Butcher LL. Transient expression of choline acetyltransferase-like immunoreactivity in Purkinje cells of the developing cerebellum. Dev Brain Res 1987; 34:303-6.

Received 12-5-1992. Revised 31-5-1993. Accepted 9-6-1993. 
Zhang Q and XW Dong

\section{PLATES}


Development of $\mathrm{AChE}$ in the rat spinal cord

\section{PLATE I}

AChE-positive strucutres stained with Tago et al procedure in the spinal cord at the following ages:

A) E14 cervical level;

B) E15 thoracic level;

C) E17 thoracic level;

D) E18 cervical; level;

E) E19 cervical level;

F) E21 cervical level. In the gray matter, AChE-positive staining appears in a ventral-to-dorsal, lateral-to-medial gradient. Note the AChE-positive staining, originally found in the position of the dorsal part of boundary cap shifted medially along the external surface of the dorsal horn and disappears by E21.

Large arrow indicates dorsal direction, small long arrow points medially. Scale bar: $100 \mu \mathrm{m}$. bc (boundary cap); DRG (dorsal root ganglion); ILN(intermediolateral nucleus); IMN(intermediomedial nucleus); LCN(lateral cervical nucleus); vh(ventral horn). 
Zhang Q and XW Dong

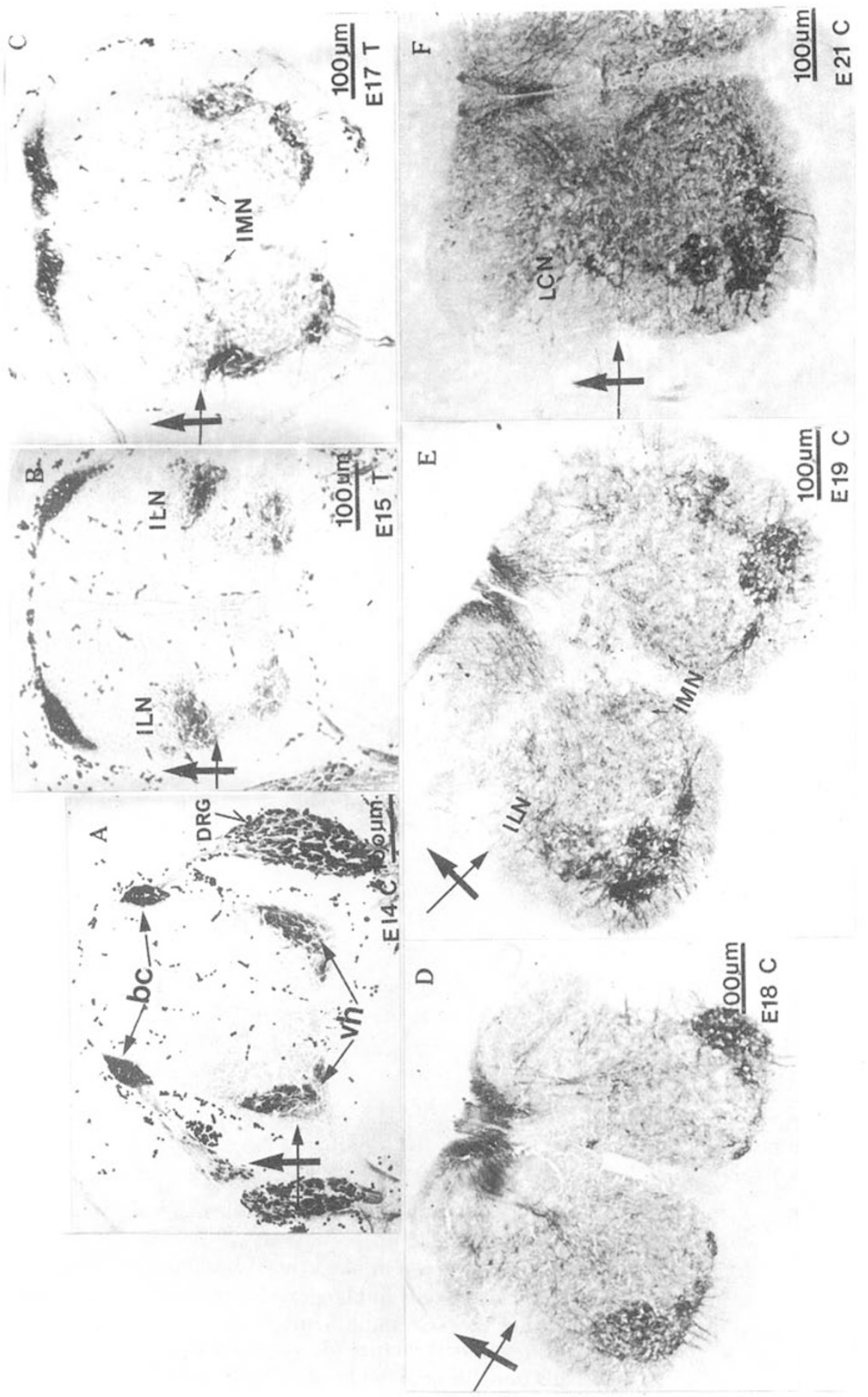


Development of AChE in the rat spinal cord

\section{Plate II}

AChE-positive cells and fibers demostrated with Mesulam modification except $\mathrm{C}$ taken from a section stained with Tago et al procedure in the spinal cord after birth.
A) AChE-positive cells in the ventral horn at cervical level at P1.
B) AChE-positive cells in the dorsal horn and lateral spinal nucleus of thoracic spinal cord at P1.
C) AChE-positive structures in the cervical spinal cord at P3.
D) AChE-positive cells in the cervical spinal cord at P5 with DFP pretreatment.
E) AChE-positive structure in the thoracic spinal cord at P7. 


\section{Zhang Q and XW Dong}

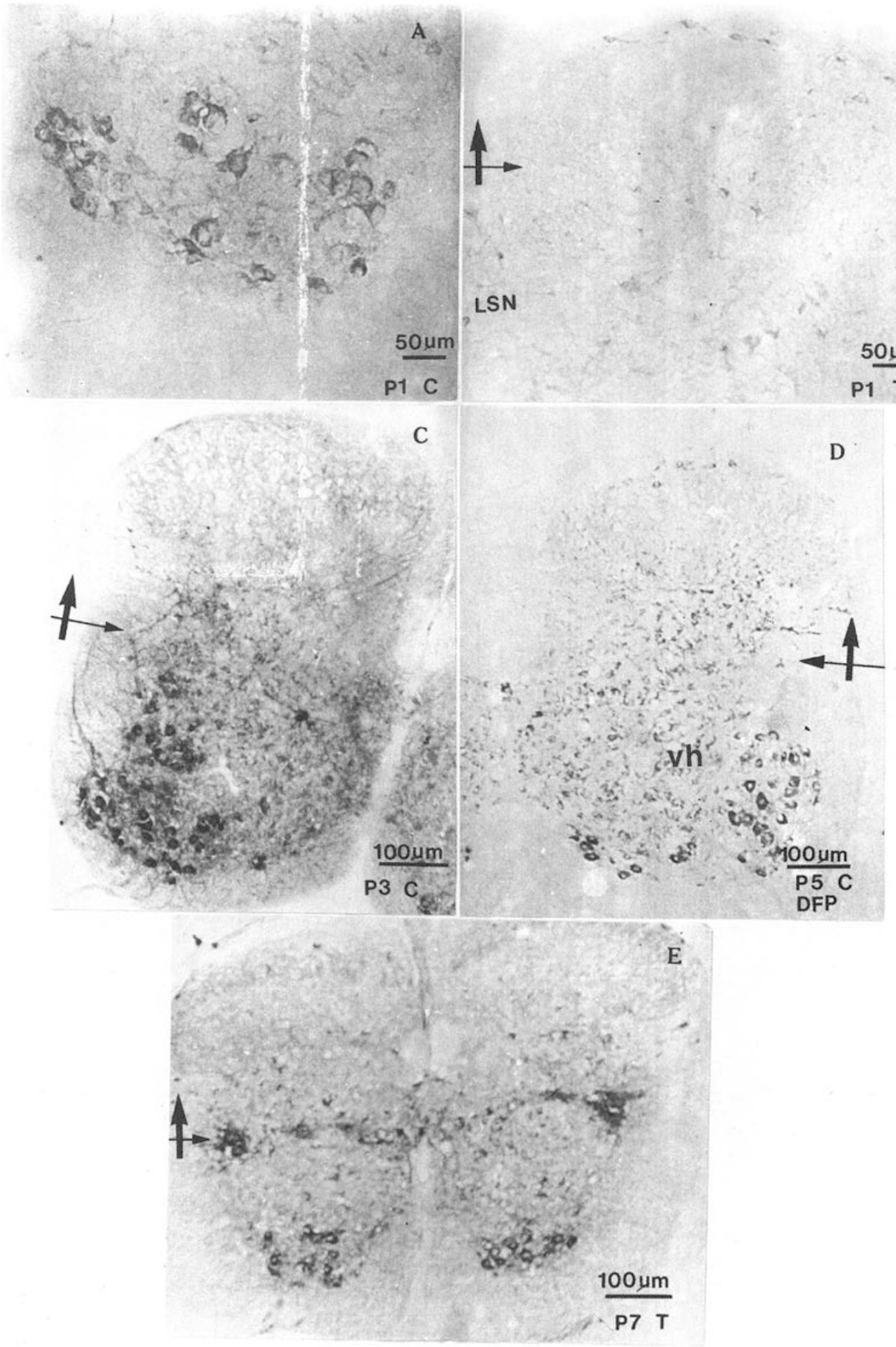


Development of AChE in the rat spinal cord

\section{Plate III}

F) AChE-positive structures in the cervical spinal cord at P10.

G) AChE-positive cells in the thoracic spinal cord at P10. with DFP pretreatment

H) AChE-positive cells in the lumbar spinal cord at P10 with DFP pretreatment.

I) AChE-positive structures in the thoracic spinal cord at P14.

J) AChE-positive cells in the thoracic spinal cord at P20 with DFP pretreatment.

K) AChE-positive structures in the thoracic spinal cord at P20.

Large arrow indicates dorsal direction, small long arrow points medically, vh(ventral horn). Scale bar:50 $\mu \mathrm{m}$ in A,B; $100 \mu \mathrm{m}$ in C-K. 
Zhang Q and XW Dong

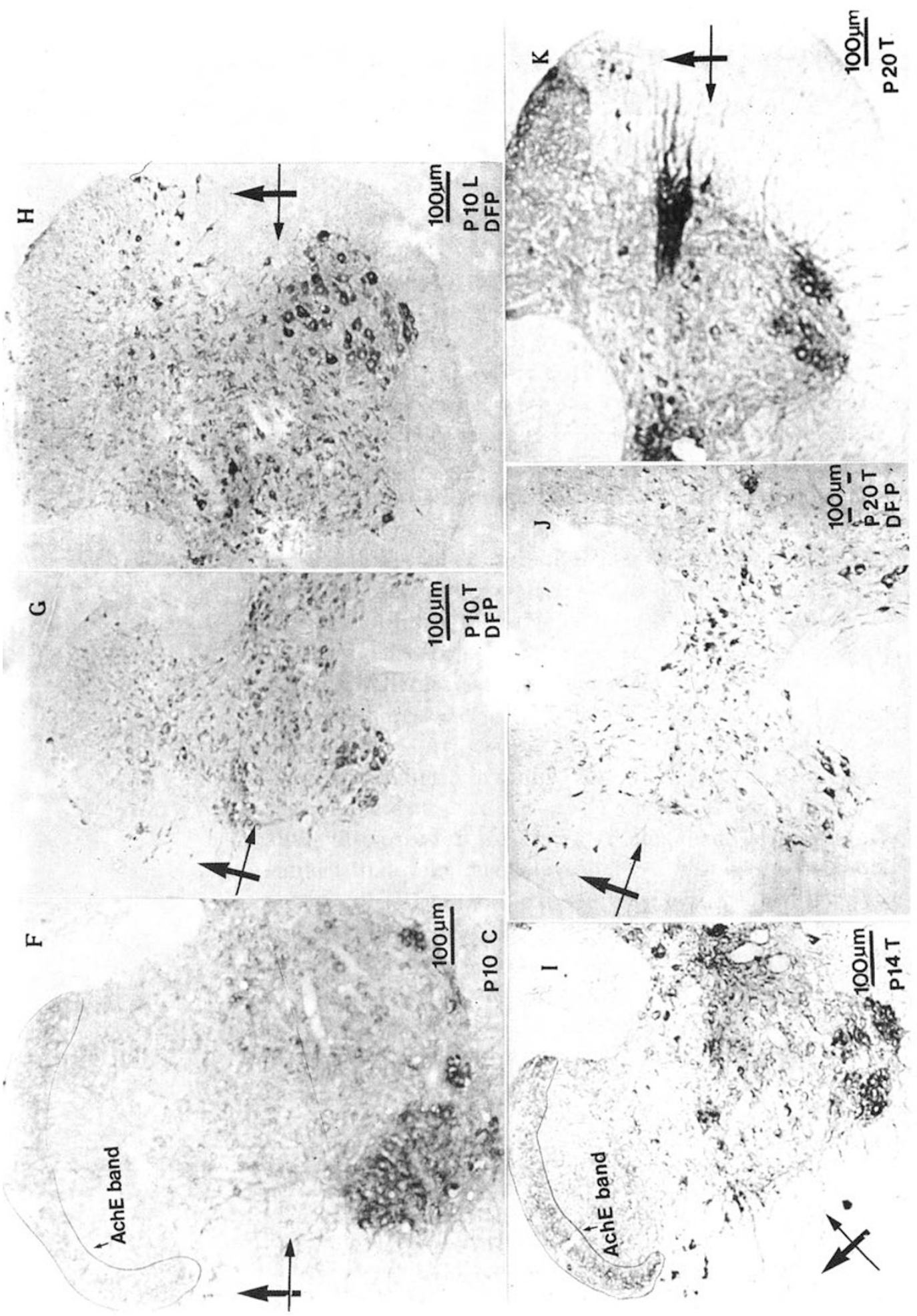

